\title{
Uso y percepción del bosque por pobladores de diferente tradición cultural de la Laguna de Mar Chiquita, Córdoba, Argentina
}

\author{
Cecilia Trillo ${ }^{1, \otimes}$; Bárbara Arias Toledo ${ }^{2} \&$ Sonia E. Colantonio ${ }^{2}$ \\ ${ }^{1}$ Departamento de Diversidad Biológica y Ecología. Facultad de Cs. Exactas, Físicas y Naturales. Univ. Nacional de Córdoba. \\ Av. Vélez Sarsfield 299, (5000) Córdoba. Argentina. ${ }^{2}$ Cátedra de Antropología. Facultad de Cs. Exactas, Físicas y Naturales. \\ Univ. Nacional de Córdoba-CONICET. Av. Vélez Sarsfield 299, (5000) Córdoba. Argentina.
}

\begin{abstract}
Resumen. El uso y la valoración de los recursos del bosque, al igual que sus servicios ecosistémicos, son percibidos de forma diferente según la filiación cultural de los pobladores. Con el objetivo de documentar estas diferencias entre "criollos" (de raigambre mestiza española-aborigen) y "gringos" (descendientes de agricultores italianos) en la zona de la Laguna de Mar Chiquita (Córdoba, Argentina) se realizaron 40 entrevistas semiestructuradas a pobladores. Se registraron 58 especies para la caza y la pesca, 18 usos diferentes del bosque y las diversas valoraciones del bosque según informantes clave. Se detectaron diferencias significativas entre los criollos y los descendientes de europeos. Los criollos mencionan más plantas medicinales, conocen el $78.3 \%$ del total de las especies para caza y el $66.6 \%$ para pesca, usan el bosque para múltiples necesidades y lo valoran de forma positiva. Por su parte, los gringos conocen el $47.3 \%$ de la diversidad disponible y valoran escasamente el bosque. Para diseñar políticas de manejo de recursos naturales es conveniente reconocer que las comunidades humanas no son homogéneas en cuanto a las necesidades de uso de recursos, en la valoración que hacen de esos recursos y en sus prácticas de manejo.
\end{abstract}

[Palabras clave: valoración, bosque chaqueño, pobladores mestizos, inmigrantes, cacería]

Aвstract. Use and perception of forest by inhabitants of different cultural tradition in Laguna de Mar Chiquita, Córdoba, Argentina. Use and assessment of forest resources and ecosystem services are differently perceived by people according to their cultural affiliation. In order to document these differences between "criollos" (Spanish-Aboriginal mestizos) and "gringos" (descendants of Italian farmers) in the Laguna de Mar Chiquita (Córdoba, Argentina), semistructured interviews to residents were conducted. A total of 58 species for hunting and fishing, 18 different uses of forest and forest valuation based on 40 key informants were recorded. Significant differences were found between "criollos" (who mentioned more medicinal plants, the use of $78.3 \%$ and $66.6 \%$ of total species for hunting and fishing, respectively, the use of the forest for multiple needs and a positive appraisement of the forest), and "gringos" (who know $47.3 \%$ of the available diversity and have little appraisement of the forest). To design policies for natural resources management it is appropriate to recognize that human communities are not homogeneous in their need of resource use, the relevance of the resources and their management practices.

[Key words: valuation, Chaco forest, creole inhabitants, hunting]

\section{INTRODUCCIÓN}

Una de las paradojas de la cultura es su carácter universal (todos los seres humanos poseen cultura) y particular a la vez, lo que implica que cada sociedad posee pautas que le son propias. Así, los seres humanos difieren no sólo en las actividades diarias y en las peculiaridades de la cultura material donde desarrollan su vida; también difieren en la percepción de lo que los rodea. Siendo la percepción la función psíquica que, luego de que el organismo capta los estímulos mediante los órganos sensoriales, interpreta las sensaciones, dándoles significado y organización (Matlin \& Foley 1996), queda claro que la percepción que cada individuo tenga del ambiente depende de su cultura y

Editor asociado: Esteban Jobbágy

$\bowtie$ ceciliatrillo1@gmail.com del "status" o rol del individuo en ésta. En este sentido, y desde el campo de la Etnobiología, Berkes (1999) define al Conocimiento Ecológico Tradicional (TEK, Traditional Ecological Knowledege), como una parte integral de la cultura local, que aporta normas o prescripciones al manejo del ambiente, posee un amplio contexto moral y ético y no separa a la naturaleza de la cultura sino que impregna a la naturaleza de elementos sagrados, incluyendo una gran cantidad de conocimiento del espacio y del tiempo.

Esta manera particular de ver el mundo se comparte y recrea entre los miembros de la comunidad mediante transmisión cultural. Tal como proponen Eyssartier et al. (2009), la transmisión cultural en comunidades rurales 
es el proceso de adquisición de conocimientos, comportamientos, actitudes, o tecnologías a través de la imitación, el aprendizaje y la enseñanza activa. Por esta razón, depende de factores como la edad, el género, el grado de aculturación y el nivel educativo, entre otros (Arias Toledo et al. 2009; Trillo 2010).

Desde su percepción de los ambientes naturales, las comunidades rurales del mundo y de Latinoamérica utilizan la vida silvestre como un recurso importante. La usan con muchos propósitos, pero en particular como fuente de alimento y de ingresos económicos (Cunningham 2001; Altricher 2006). Tal como lo registra el último informe de la situación de los bosques de la FAO (2011), los productos alimentarios, los animales vivos y los exudados son los principales productos forestales no maderables extraídos de los bosques de América Latina y del Caribe. Por su parte, la recolección de estos productos la realizan personas que dependen de los bosques. En Argentina, trabajos con productores ganaderos de las Salinas Grandes en Catamarca registran esta relación con el bosque, y señalan que es importante la caza de fauna silvestre, la recolección de miel y de otros productos naturales para alimentación y uso medicinal de las familias, junto con la extracción de productos forestales para leña y construcciones rurales (Cavanna et al. 2009). En Córdoba, Trillo et al. (2014) enumeran 15 usos diferentes del bosque realizados por pobladores criollos del Bosque Chaqueño Occidental, quienes alimentan su ganado y conservan la salud de su familia con recursos boscosos.

En relación a la caza de especies silvestres, los trabajos de Barbarán (1999, 2003), Arenas (2003) y Arenas \& Poirini (2009) revelan que la cacería de fauna silvestre puede ser un medio de subsistencia para comunidades originarias y criollas. La sistematización realizada por Pautasso (2003) en la provincia de Santa Fe detectó 40 especies silvestres cazadas para subsistencia. En Córdoba, los trabajos de Navarro et al. (1997), con prácticas de manejo sustentabledeLagostomusmaximus(Desmarest 1817) en zonas agrícolas, y el aporte de Berduc (2004) revelan acciones cinegéticas sobre 15 especies en Bosque Serrano. Sin embargo, existe poca información acerca del consumo de animales en los ambientes naturales del noreste de la provincia, a excepción del uso como medicinales sistematizado por Arias Toledo \& Trillo (2014), que registra 10 especies utilizadas con fines medicinales.
En trabajos anteriores realizados en el Bosque Chaqueño Occidental de Córdoba con comunidades ganaderas campesinas autodenominadas criollos por Trillo et al. (2014) se registraron 123 especies con 15 usos diferentes que satisfacen distintas necesidades, algunas vitales y otras inmateriales. Estos pobladores de origen mestizo aborigenespañol poseen una valoración positiva del bosque, sin distinción de oficios, edad o sexo ya que es el espacio vital para desarrollar su vida de ganaderos, que les permite mantener su identidad como criollos y heredar sus tradiciones a sus hijos (Trillo 2010). Sin embargo, el Bosque Chaqueño Oriental de Córdoba es heterogéneo en cuanto a orígenes humanos y se encuentra conformado entre otros por el grupo mas antiguo de mestizos aborigen-español y los nuevos actores sociales que llegan en el Siglo XX. Este nuevo grupo se formó a partir de 1900 por inmigrantes italianos y españoles; hasta el presente se mantienen como subgrupos diferenciales mediante pautas de cruzamiento particulares (Ríos Villamil 2013). En nuestros días, ofrece un mosaico genético y cultural.

Considerando que para los gestores de recursos naturales es un desafío encontrar un balance entre conservación y uso que no comprometa la persistencia de las especies ni la supervivencia del poblador local, y teniendo en cuenta que la pertenencia a diferentes tradiciones culturales definirían percepciones y usos de los ambientes naturales disímiles, el presente trabajo se desarrolla bajo la hipótesis de que la valoración, el número de especies utilizadas y la diversidad de usos será diferente según el origen sociocultural del poblador. Por ello se describirá la biodiversidad comparada utilizada en caza, pesca y recursos vegetales según la filiación cultural declarada por cada poblador, a la vez de identificar las valoraciones que cada subgrupo posee de los ambientes boscosos.

\section{Materiales y Métodos}

A fin de establecer las diferencias entre los subgrupos poblacionales autodefinidos como criollos y gringos, en relación a las valoraciones, prácticas y biodiversidad que utilizan se realizaron 40 entrevistas (20 a cada subgrupo) a pobladores de ambos sexos, de entre 30 y 60 años de 8 localidades de la zona de estudio. Se realizó una muestra no aleatoria incluyendo sólo informantes clave, que son aquellos especialistas o expertos nativos que tienen un conocimiento profundo de un aspecto particular de la cultura local (Costa- 
Neto 2002), y de tal forma son reconocidos por el resto de la comunidad. El método de muestreo fue el de bola de nieve. Esta estrategia resulta útil como una respuesta para resolver los problemas asociados con el muestreo en poblaciones aisladas (Aguilera et al. 2003).

Las encuestas fueron realizadas en poblaciones de la zona sur de Laguna Mar Chiquita (Miramar, Marull, La Para, Balnearia), de la zona oeste-noroeste (Sebastián El Cano, Villa Candelaria, Guanaco Muerto), y del este (Villa Trinidad- Santa fe) (Figura 1), e incluyeron preguntas abiertas acerca de su vida en relación al bosque, de su percepción y perspectivas en conservación, así como interrogantes tendientes a lograr una auto-caracterización dentro de algún grupo social. Asimismo, mediante preguntas semi-estructuradas se recabó información acerca de la flora y fauna utilizada. Se indagó sobre la principal fuente de ingresos (e.g., cría de animales, caza, pesca, agricultura, mantenimiento de huerta, usos del bosque). Se preguntó a los miembros de la familia que desarrollan esas actividades, qué técnicas e instrumentos utilizan, en qué paisajes los llevan adelante, cuáles son las especies consumidas y los criterios de reparto entre miembros familiares o de venta de lo obtenido.

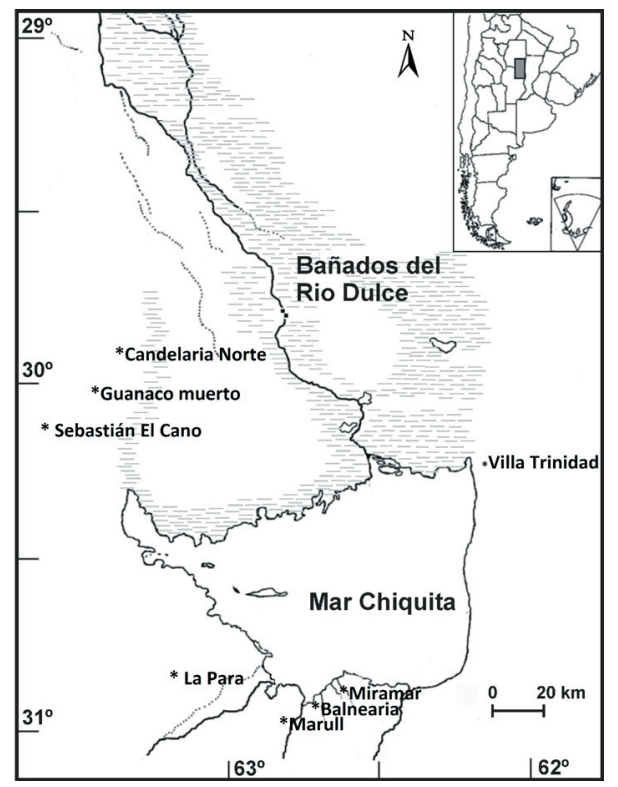

Figura 1. Ubicación de las localidades de estudios en la Laguna Mar Chiquita (Miramar, Marull, La Para, Balnearia), de la zona oeste-noroeste (Sebastián El Cano, Villa Candelaria, Guanaco Muerto), y del este (Villa Trinidad, Santa Fe), Argentina.

Figure 1. Map of locations of studies at the Laguna Mar Chiquita (Miramar, Marull, La Para, Balnearia) westnorthwest (Sebastián El Cano, Villa Candelaria, Guanaco Muerto), and east (Villa Trinidad, Santa Fe), Argentina.
Las especies vegetales que no pudieran ser identificadas "in-situ" fueron fotografiadas, colectadas y herborizadas para ser cotejadas luego con herbarios de referencia (Cecilia Trillo, colección personal). En primera instancia, las especies animales fueron mencionadas por sus nombres comunes y descriptas en los cuestionarios abiertos iniciales. Con tal información, un especialista las identificó y generó un muestrario fotográfico (cuando se tratara de animales silvestres). Ese muestrario sirvió para que en las entrevistas siguientes los pobladores señalaran con certeza a las especies implicadas. En el caso de que el entrevistado poseyera materiales de origen animal, eran fotografiados o colectados y de cada especie señalada se solicitó una descripción detallada a nivel morfológico y de hábitos, para incrementar el grado de certeza de la identificación. En el caso de tratarse de animales domésticos que se poseyeran en el lugar, fueron identificados in-situ. Toda la información obtenida fue cotejada y verificada por un zoólogo especializado en el área de estudio.

Los datos obtenidos se analizaron de la siguiente forma: descripciones cualitativas y comparativas acerca de percepciones, prueba de Fisher para estimar las diferencias en la diversidad de usos realizados entre subgrupos, prueba de $\mathrm{T}$ apareada para diferencias de medias, a fin de establecer diferencias en el número de plantas medicinales utilizadas por subgrupo, análisis de varianza (ANOVA) para determinar diferencias en el número de especies animales aprovechadas por subgrupo. Finalmente, se realizó una revisión bibliográfica para referenciar el uso de las especies citadas por folcloristas, zoólogos y etnobiólogos; para ello se consultó Di Lullo (1946), Arenas (2003), Pautasso (2003), Altricher (2006), Haro et al. (2006) y Torres et al. (2006), Arenas \& Porini (2009).

\section{Área de estudio}

La vegetación que ocupa la cuenca sin desagüe de Mar Chiquita es compleja y variada. Su diversidad y distribución espacial están determinadas por la interacción entre el relieve y la hidrología. Según Mengui et al. (2006) y Curto (2009), en la zona el bosque chaqueño típico dominado por Aspidosperma quebracho-blancoschltdl.ZZiziphusmistolGriseb.

y Prosopis sp. ocupan las áreas sin suelo salino, mientras que en matorrales bajos de especies halófitas se presentan arbolitos xerófilos de Allenrolfeas spp., Acacia aroma, Geoffroea 
decorticans (Gillies ex Hook. \& Arn.) Burkart, GrawbowskiaduplicataArn.,Maytenusvitis-idade Griseb. y Prosopis strombulífera (Lam.) Benth; la zona este se caracteriza por la presencia de Trithrinax campestris (Burmeist.) Drude \& Griseb., Prosopis algarrobilla Griseb., Cereus validus Haw. y varias especies de arbustos del género Acacia. Próximos a la desembocadura de los ríos Primero y Segundo hay bosquecillos ribereños de Celtis spinosa Spreng., Salix humboldianaWilld.ySapiumhaematospermun Mull. Arn.

Con respecto al estado de conservación (Menghi 2006; Curto 2009) es posible afirmar que en los Bañados del Río Dulce, la intervención humana más importante se basa en la ganadería extensiva (bovina, ovina y equina), permanente en áreas inundables y estacional en aquellas sujetas a la influencia fluvial periódica.

En relación a la fauna cabe señalar que, de acuerdo a Haro et al. (2006), la ictiofauna de la laguna es muy pobre dado que la alta salinidad de sus aguas es una limitante clave para los peces. En cambio, los bañados del Río Dulce albergan la mayor riqueza de peces de la reserva y, de manera similar, posee una mayor abundancia de mamíferos y aves (Torres et al. 2006).

En relación a las características socioculturales de los pobladores, la mayoría de los entrevistados se autodefinieron como criollos o como gringos. Es preciso aclarar que los subgrupos no fueron definidos a priori por los investigadores, sino que surgieron como referencias de los mismos pobladores, y en la clasificación siempre se respetaron los grupos a los que ellos decían pertenecer.

\section{Resultados}

En primera instancia, al haber surgido la diferenciación en dos subgrupos poblacionales se profundizó en los factores que determinan el sentimiento de pertenencia a uno $u$ otro grupo. Así, mientras los gringos se autodefinen y son definidos por el otro como agricultores, destacan que no realizan actividades tradicionales de subsistencia tales como la caza y la pesca (aunque sí lo hacen como deporte), y son heterogéneos en su percepción de los bosques nativos; la población se reparte en partes iguales entre los que creen que debe ser protegido, los que no sienten interés al respecto y los que tienen una percepción negativa y creen que deben reconvertirse todas las áreas boscosas en tierra de cultivo. Los criollos serían ganaderos, con apego a actividades de subsistencia y con una percepción positiva del bosque.

En la Tabla 1 se observa la cantidad de especies diferentes que se mencionan para la diversidad de usos registrados para los recursos silvestres, organizados en forma comparativa entre gringos y criollos. Los usos reflejan claramente la utilización diferencial del bosque por parte de pobladores con diferente filiación cultural. De los 18 usos extractivos registrados, 12 son exclusivamente mencionados por criollos y, en los 5 usos compartidos por los dos grupos humanos, es mayor la cantidad de citas de especies por los criollos en un orden del triple o cuádruple (prueba de Fischer, $P=0,0001$ ).

Existen diferencias significativas en el uso de las especies medicinales del bosque. Los criollos son los que mencionan y utilizan mayor número de especies $(t=8.39$ $\alpha=0,000$; media de criollos $=1.667$ y media de gringos $=0.317$; coeficiente de variación para gringos $=2.40$ y para criollos $=0.74$ ). También se encontraron diferencias significativas en el consenso (número de veces) que es citada cada especies que cazan y pescan según filiación cultural (ANOVA, $P=0.026)$; media para criollos $=9.47$ y media para gringos $=5.22$.

Tabla 1. Cantidad de especies para diferentes usos que se satisfacen con el bosque, según la filiación cultural de los pobladores "criollos" y "gringos" en la zona de influencia de los Bañados del Río Dulce y la Laguna de Mar Chiquita.

Table 1. Number of species for different uses are satisfied with the forest as the cultural affiliation of the people "criollos" and "gringos" in the Bañados del Río Dulce and Lagoon of Mar Chiquita

\begin{tabular}{lcc}
\hline \multicolumn{1}{c}{ Usos } & $\begin{array}{c}\mathrm{N}^{\circ} \text { de sp } \\
\text { citadas por } \\
\text { "criollos" }\end{array}$ & $\begin{array}{c}\mathrm{N}^{\mathrm{o}} \text { de sp } \\
\text { citadas por } \\
\text { "gringos" }\end{array}$ \\
\hline Garrote para cazar & 4 & 0 \\
Elaboración de carbón & 4 & 0 \\
Cercos para el ganado & 1 & 0 \\
Alambrados & 1 & 0 \\
Vivienda & 2 & 0 \\
Herramientas & 4 & 0 \\
Tintes naturales & 2 & 0 \\
Clarificar agua & 1 & 0 \\
Veterinarias & 2 & 0 \\
Veneno & 1 & 0 \\
Mascotas & 3 & 0 \\
Sombra & 1 & 0 \\
Alimenticias & 9 & 3 \\
Medicinales & 78 & 4 \\
Forrajeras & 4 & 1 \\
Protectoras & 3 & 1 \\
Leña & 4 & \\
Caza & exclusivas & \\
& & 16 \\
\hline
\end{tabular}


Tabla 2. Listado de nombres comunes y científicos de especies citadas para caza y pesca en la zona de influencia de los Bañados del Río Dulce y Laguna de Mar Chiquita. Número de pobladores que citan la especies según filiación cultural criollos/gringos. *Citado por poblador finlandés.

Table 2. Scientific and common names cited for hunting and fishing in the area of influence of the Rio Dulce and Bañados Laguna Mar Chiquita. Number of inhabitants mentioning the species according to cultural affiliation, Creole / gringos cultural affiliation. ${ }^{*}$ Cited by Finnish resident.

\begin{tabular}{|c|c|c|}
\hline Familia/Nombre científico & Nombre común & $\begin{array}{l}\mathrm{N}^{\mathrm{o}} \text { de menciones por } \\
\text { filiación cultural criollos / } \\
\text { gringos }\end{array}$ \\
\hline Fam. Anatidae & & \\
\hline Coscoroba coscoroba (Molina,1782) & Ganso salvaje & 1 \\
\hline & Pato & $1 / 1$ \\
\hline Fam. Boidae & & \\
\hline $\begin{array}{l}\text { Boa constrictor occidentales (Philippi, 1873) } \\
\text { Fam. Canidae }\end{array}$ & Lampalagua & $4 / 1$ \\
\hline Cerdocyon thous (Linnaeus, 1766) & Maigato & 2 \\
\hline Chrysocyon brachyurus (Illiger, 1815) & Aguará guazú & 3 \\
\hline Pseudalopex gymnocercus (Fischer, 1814) & Zorro gris & $3 / 2$ \\
\hline Fam. Cariamidae & & \\
\hline $\begin{array}{l}\text { Chunga burmeisteri (Hartlaub, 1860) } \\
\text { Fam. Caviidae }\end{array}$ & Chuña & $1 / 1$ \\
\hline Dolichotis salinicola (Burmeister, 1876) & Conejo de los palos & $1 / 2$ \\
\hline Galea musteloides (Meyen, 1832) & Cuis & $2 / 1$ \\
\hline $\begin{array}{l}\text { Hydrochoerus hydrochareis (Linnaeus,1766) } \\
\text { Fam. Cervidae }\end{array}$ & Carpincho & 4 \\
\hline Mazama gouazoubira (G. Fischer, 1814) & Corzuela & $8 / 3$ \\
\hline Fam. Ciconiidea & & \\
\hline $\begin{array}{l}\text { Ciconia maguari (Gmelin, 1789) } \\
\text { Fam Cracidae }\end{array}$ & Cigüeña & * \\
\hline Ortalis canicollis (Wagler, 1830) & Charata & 1 \\
\hline Fam. Chinchillidae & & \\
\hline Lagostomus maximus (Desmarest, 1817) & Vizcacha & $10 / 4$ \\
\hline Fam. Columbidae & Paloma & 2 \\
\hline $\begin{array}{l}\text { Zenaida auriculata (Des Murs, 1847) } \\
\text { Fam. Dasypodidae }\end{array}$ & Torcaza & 1 \\
\hline Chaetophractus spp. (Fitzinger, 1871) & Quirquincho & 5 \\
\hline Chaetophractus vellerosus (Gray, 1865) & Mulita & 2 \\
\hline Fam. Didelphydae & & \\
\hline Didelphys albiventris (Lund, 1840) & Comadreja & 3 \\
\hline Fam. Felidae & & \\
\hline Leopardus geoffroyi (d'Orbigny Gervais, 1844) & Gato montés o barcino & $0 / 1$ \\
\hline Leopardus colocolo (Molina, 1782) & Gato de los pajonales & * \\
\hline Puma concolor (Linneo, 1771) & Puma & $4 / 1$ \\
\hline $\begin{array}{l}\text { Puma yagouaroundi (Étienne Geoffroy Saint-Hilaire, 1803) } \\
\text { Fam. Leporidae }\end{array}$ & Gato negro & * \\
\hline $\begin{array}{l}\text { Lepus (Eulagos) europaeus (Pallas, 1778) } \\
\text { Fam. Leptodactylidae }\end{array}$ & Liebre & $5 / 3$ \\
\hline Leptodactyls spp. (Fitzinger,1826) & Rana & 1 \\
\hline $\begin{array}{l}\text { Fam. Mephitidae } \\
\text { Conepatus chinga (Molina,1782) }\end{array}$ & Zorrino & $2 / 1$ \\
\hline Fam. Mustelidade & & \\
\hline Galictis cuja (Molina, 1782) & Hurón & 1 \\
\hline Lontra longicaudis (Olfers, 1818) & Lobito de río & * \\
\hline Fam. Myocastoridae & & \\
\hline Myocastor coypus (Molina, 1782) & Nutria, coipo & $2 / 2$ \\
\hline Fam. Phalacrocoracidae & & \\
\hline Phalacrocorax brasilianus (Gmelin,1789) & Bigua & * \\
\hline Fam. Phoenicopteridae & & \\
\hline Phoenicopterus chilensis (Molina,1782) & Flamenco & * \\
\hline $\begin{array}{l}\text { Fam. Podicipedidae } \\
\text { - }\end{array}$ & Maca & * \\
\hline Fam. Pstitacidae & & \\
\hline $\begin{array}{l}\text { Thectocercus acuticaudata (Vieillot, 1818) } \\
\text { Fam. Rheidae }\end{array}$ & Loro & 1 \\
\hline Rhea americana (Linnaeus, 1758) & Ñandú & $4 / 1$ \\
\hline Fam. Suidae & & \\
\hline Sus scrofa (Linnaeus, 1758) & Jabalí, chancho & $6 / 4$ \\
\hline Fam. Tayassuidae & & \\
\hline Pecari tajacu (Linnaeus, 1758) & Pecari & $7 / 2$ \\
\hline
\end{tabular}


Tabla 2: Continuación

Table 2: Continuation

\begin{tabular}{|c|c|c|}
\hline Familia/Nombre científico & Nombre común & $\begin{array}{l}\mathrm{N}^{\mathrm{o}} \text { de menciones por } \\
\text { filiación cultural criollos / } \\
\text { gringos }\end{array}$ \\
\hline Fam. Teiidae & & \\
\hline $\begin{array}{l}\text { Tupinambis rufescens (Günther, 1871) } \\
\text { Fam.Testudinidae }\end{array}$ & Iguana & $6 / 2$ \\
\hline Chelonoidis chilensis (Gray, 1870) & Tortuga de tierra & 2 \\
\hline Fam. Tinamidae & Tortuga de agua & 1 \\
\hline Eudromia elegans (Geoffroy Saint-Hilaire, 1832) & Martineta o copetona & $3 / 2$ \\
\hline Nothoprocta cinerascens (Burmeister,1860) & Perdiz montaraz & $2 / 2$ \\
\hline Nothura maculosa (Temminck, 1815) & Perdiz & $5 / 4$ \\
\hline $\begin{array}{l}\text { Rhynchotus rufescens (Temminck, 1815) } \\
\text { Fam. Tytonidae }\end{array}$ & Perdiz ala colorada & $1 / 2$ \\
\hline $\begin{array}{l}\text { Tyto alba (Scopoli, 1769) } \\
\text { Fam. Viperidae }\end{array}$ & Lechuza & 2 \\
\hline $\begin{array}{c}\text { Crotalus durissus (Linnaeus, 1758) } \\
\text { Pesca }\end{array}$ & Cascabel & 1 \\
\hline Fam. Anastomidae & & \\
\hline $\begin{array}{l}\text { Leporinus obtusidens (Valenciennes, 1847) } \\
\text { Fam. Atherinopsidae }\end{array}$ & Boga & 1 \\
\hline $\begin{array}{l}\text { Odontesthes bonariensis (Valenciennes, 1835) } \\
\text { Fam. Characidæ }\end{array}$ & Pejerrey & $2 / 5 *$ \\
\hline Cheirodon interruptus (Jenyns, 1842) & Mojarritas & $1 / 1$ \\
\hline Oligosarcus jenynsii (Günther, 1864) & Dientudos & * \\
\hline $\begin{array}{l}\text { Salminus maxillosus (Cuvier, 1816) } \\
\text { Fam. Cyprinidae }\end{array}$ & Dorado & * \\
\hline $\begin{array}{l}\text { Cyprinus Carpio (Linnaeus, 1758) } \\
\text { Fam. Erythrinidae }\end{array}$ & Carpa, overo & $4 / 2 *$ \\
\hline $\begin{array}{l}\text { Hoplias malabaricus (Bloch, 1794) } \\
\text { Fam. Pimelodidae }\end{array}$ & Tararira & $1 / *$ \\
\hline $\begin{array}{l}\text { Pimelodus albicans (Valenciennes, } 1840 \\
\text { Fam. Prochilodontidae }\end{array}$ & Moncholos & $5 / 2$ \\
\hline $\begin{array}{l}\text { Prochilodus lineatus (Valenciennes, 1836) } \\
\text { Fam. Synbranchidae }\end{array}$ & Sábalo & $1 / 1 *$ \\
\hline Synbranchus marmoratus $\left(\right.$ Bloch $\left._{\llcorner} 1795\right)$ & $\begin{array}{l}\text { Anguilas } \\
\text { Ostra } \\
\text { Cangrejo } \\
\text { Carpa rosada }\end{array}$ & $\begin{array}{l}2 \\
* \\
* \\
*\end{array}$ \\
\hline
\end{tabular}

En la Tabla 2 se detallan las especies mencionadas para caza y pesca. El bosque es el sitio donde se encuentra la mayoría de las especies de caza, que incluye la utilización de más de 46 especies animales (21 mamíferos, 17 aves, 5 reptiles y 1 anfibio). Asimismo, se registró el uso de 12 especies de peces y 2 de invertebrados que son posibles encontrar en los ríos Xanaes, Suquía, Dulce, y en la Laguna de Mar Chiquita.

Esta diversidad no es utilizada por igual por ambos subgrupos. Los criollos cazan y utilizan más de 46 especies (20 de las cuales son citadas sólo por informantes de ese origen). Los gringos citan 25 especies, de las cuales sólo de una no se registró uso por parte de criollos: el hurón (que no es utilizada para la alimentación sino para mantenerlo vivo y que realice el control de roedores en el domicilio). En relación a la pesca, de las 13 especies mencionadas como utilizadas, 3 son exclusivas de los criollos. Cabe mencionar que un poblador de origen finlandés que no se autodefinió en ningún grupo, mencionó 12 especies de manera exclusiva. De las 58 especies citadas para caza y pesca, 57 se encuentran documentadas por la bibliografía etnobiológica y folclórica (Di Lullo 1946; Arenas 2003; Pautasso 2003; Arenas \& Porini 2009), con excepción de los pichones de lechuza (una especie que además de no aparecer mencionada como cinegética, es temida por ser una "señal de mal agüero").

La especie más citada por ambos subgrupos es la vizcacha, que actualmente no es fácil de encontrar según los comentarios de los pobladores. Es interesante considerar que este roedor es una plaga para la agricultura, y que ha sido perseguida en los últimos 50 años, en paralelo con la profunda transformación del bosque en campos de cultivo en la zona de estudio (Rosacher 2011). En orden decreciente, mencionan la corzuela, el pecarí, las iguanas y el jabalí. Salvo esta última especie, que ha ampliando su distribución geográfica en cada década, los tres primeros se encuentran en franca regresión numérica (Barbarán 1999). 
Este hecho ha sido detectado por los cazadores, quienes expresan que "cada vez hay que ir más lejos a buscarlos" y que "la cantidad de especies e individuos se ha reducido mucho en la actualidad". Entre otros factores, mencionan la disminución de bosque y el aumento de terrenos explotados para agricultura.

Con respecto a las prácticas y al valor comercial, se pudieron detectar diferencias notables entre la caza comercial y la caza para subsistencia o consumo familiar. La primera es realizada por los gringos, con la utilización de armas de fuego y perros entrenados, y en ocasiones en el marco de excursiones de caza para turistas. Las especies animales cazadas bajo esta modalidad son minoría (de 5 a 8 , básicamente diferentes especies de Tinámidos [perdices]), y lo obtenido suele utilizarse como trofeo (se reparte entre los miembros de la excursión); en ocasiones se realizan escabeches y en sólo un caso se comercializaban en conserva. En cada excursión de caza se obtienen tantos animales como sea posible. En cambio, la caza para consumo familiar es realizada por criollos e involucra a la totalidad de las especies relevadas. Se realiza ocasionalmente con armas de fuego y trampas caseras (e.g., ganchos de metal unidos a cañas de madera para extraer los "pichones" de comadreja [Didelphys albiventris] del nido), y sólo se caza lo que se consumirá (de 1 a 5 animales, dependiendo de su tamaño. Por ejemplo, sólo una corzuela o pecarí, pero pueden ser cuatro o cinco perdices); se aprovecha la totalidad de lo obtenido. La caza para consumo familiar es realizada en la actualidad en pocas ocasiones al año, aunque algunas familias recuerdan que hasta hace diez o quince años era la forma común de obtención de proteínas animales.

En relación a las expresiones que reflejan su percepción y valoración del bosque, existen diferencias entre los dos subgrupos. El $100 \%$ de los pobladores criollos expresa una valoración positiva del bosque, considerándolo como el sitio de donde se obtienen bienes y servicios (e.g., regula el clima y protege de su inclemencias, etc.) y como espacio de trabajo (ganadería). Todo esto desaparece al convertirse a la agricultura. Se asocia la pérdida del bosque a la desaparición de tradiciones: "Los animales se van por culpa de las fumigaciones y porque no tienen monte"; "El campo era para todos, en cambio, la siembra es para los ricos nomás". Expresan su preocupación y deseo de conservación de la áreas boscosas existentes: "Ya está bien, no se debe sacar más". En cambio, los gringos dividen su expresiones en partes iguales entre aquellas positivas ("Lo más lindo"; "Por algo Dios puso las plantas"; "Ya se pasó la cantidad de monte que se podía talar"), valoraciones intermedias (gusta la "chacra" y que esté "limpio", pero debería quedar algo de monte para "mantener las tradiciones"), como así también valoraciones negativas en las que el monte es percibido como sucio, peligroso y sitio de grandes esfuerzos ("Ahora está más lindo porque está limpio"; "Las casas tienen que estar abiertas"; "El monte no sirve para nada").

\section{DISCUSIÓN}

En primera instancia, se destaca que pertenecer o sentirse parte del grupo de origen criollo o gringo es uno de los principales factores que definen la cantidad de conocimiento etnobiológico que posee un poblador y el grado de acercamiento con el bosque. Tal autopercepción del criollo como ganadero y del gringo como agricultor ha sido previamente descripta por historiadores, que relatan que durante la primitiva inmigración española (que diera origen a los actuales criollos) fue presupuesto fundamental tomar distancia de la gente de la tierra, teniendo como premisa básica "de todo menos labriego", siendo la apariencia de la condición que habían creído alcanzar (Corcuera 2006). Así, el trabajo de las primitivas familias fue criar ganado y arriarlo, mientras que los más pobres pasaron a la temida condición de labriego. Por el contrario, siendo la inmigración italiana muy posterior, el panorama mundial y local era diferente y el acceso a una porción de tierra que pudiera ser trabajada resultó completamente aceptable y trampolín de progreso para muchas familias.

Queda en evidencia que los pobladores que viven en la zona de influencia de la Mar Chiquita y los Bañados del río Dulce poseen un refinado conocimiento de las especies que se presentan en su entorno, particularmente si su origen o grupo de filiación es de los criollos, tal como lo describieron en el pasado para pobladores de Santiago del Estero, el folclorista Di Lullio (1946) y Trillo et al. (2014) para pobladores del oeste de Córdoba. Estudios similares en Salta realizados por Barbarán (2003) con criollos y aborígenes Wichis, obtuvo una lista de 45 especies cazadas con fines de subsistencia, comercial y deportiva con similares percepciones sobre estos recursos. En bosques tropicales de México, Didac et al. 
(2013) con pobladores nativos revelan usos similares del recurso faunístico; presenta una lista de 46 especies de vertebrados utilizados como fuente de subsistencia por comunidades de descendencia maya en la selva Lacandona, con prácticas ancestrales de caza, similares en cuanto a la intención de la cacería y el reparto de carne del monte.

Estas prácticas de pautas de aprovechamiento registradas muestran una continuidad en el tiempo de los criollos y aborígenes, ya que, tal como lo expresan Medrano \& Rosso (2012), muchas de las especies mencionadas formaban parte del recurso cinegético de poblaciones guaicurúes desde épocas prehispánicas, existiendo asimismo relatos acerca de cazadores santiagueños especializados en los recursos del Gran Chaco.

Considerando los conceptos aquí expuestos, observamos que los miembros del subgrupo criollo perciben el bosque como un espacio para satisfacer necesidades múltiples, que involucra no sólo aspectos económicos (como mantener bien alimentado su ganado), sino también de satisfacciones emocionales. Al respecto, Trillo et al. (2014) proponen que sin importar la actividad económica que desarrollen, el género o la edad de los entrevistados, los resultados sugieren que todos los pobladores criollos valoran de manera positiva el monte que los rodea. Similares resultados reportan Majumdar et al. (2008) y Karppinen (1998), en el sentido de que la mayoría de los pobladores valora el bosque como un sitio para resolver múltiples propósitos económicos y emocionales.

Los resultados presentados en este estudio reflejan al menos dos maneras diferentes de conocer e interpretar los ambientes naturales que traen como consecuencia dos estrategias diferentes de gestionar dichos ambientes. Tal como propone Berkes (1996), este conocimiento se analiza en distintos niveles: el conocimiento de las especies, el conjunto de técnicas y prácticas que suponen la comprensión de procesos ecológicos, el conjunto de reglas y códigos sociales que regulan el uso, y el conjunto de percepciones e interpretaciones que realizan sobre un ambiente. Estas dos miradas disímiles de interpretar el ambiente natural explicarían, entre otros factores, el proceso de deforestación y transformaciones profundas que se registran en la zona de estudio desde los últimos 150 años y que se ha acelerado en las dos décadas pasadas.

En relación al uso de las plantas, trabajos realizados sobre el uso de plantas medicinales y los cambios del uso del suelo (Arias Toledo et al. 2010,2014) revelaron una clara asociación entre el conocimiento de las especies vegetales medicinales y la proporción de bosque nativo en el paisaje. La mención de especies nativas con respecto a las totales disminuyó a medida que los bosques se reducen. Lo mismo puede verse en la correlación, que asocia de manera significativa el aumento en el uso de especies medicinales exóticas con el aumento del cultivo intensivo del suelo. El avance de la frontera agrícola no sólo impacta sobre el ambiente natural y sobre la cultura de los pobladores a través de la erosión del conocimiento tradicional, es posible que haga desaparecer de la memoria colectiva de pobladores las formas de manejo tradicionales (que son más sustentables que el monocultivo industrial). Esto agrava tanto la situación ambiental de la región como la situación socioeconómica de los pobladores (Arias Toledo et al. 2010).

Estos cambios afectan de manera desigual a los dos subgrupos estudiados, ya que el tipo de explotación del bosque por parte de los criollos es del tipo de subsistencia. En cambio, los gringos solo usan el bosque para salir a caza como actividad recreativa y poseen, en general, actividades productivas asociadas a la agricultura o al comercio. Al respecto, Giraudo (2009) concluye que la defaunación tendrá consecuencias indeseables, generando cambios en las biotas y ecosistemas, que repercutirán directa e indirectamente sobre distintos aspectos socioeconómicos, culturales y políticos. Esto afectará más profundamente a sectores de la población en situaciones de pobreza y con mayor dependencia de los recursos naturales para su subsistencia, como lo son muchos campesinos criollos y aborígenes. En este sentido, su desaparición, habitualmente para su reconversión para agricultura intensiva, es percibida en forma negativa, asociada a un sentimiento de pérdida y desarraigo en la medida que ya no pueden realizar sus actividades tradicionales $\mathrm{y}$, en ocasiones, se ven obligados a migrar a zonas urbanas o periurbanas. Considerando que la autopercepción de los pobladores (base de la construcción de la identidad cultural) es en relación a los bosques, y que de ellos se obtiene una variedad de productos (que, aprovechados mediante técnicas tradicionales serían sustentables a lo largo de los siglos Trillo et al. [2014]), la conservación de biodiversidad y de conocimientos tradicionales asociados al bosque debería ser asumida tanto por razones pragmáticas como por el cumplimiento 
de nuestra responsabilidad de conservar el patrimonio cultural de generaciones pasadas.

Agradecimientos. A todos los pobladores que compartieron sus experiencias de vida. Al Dr. Ricardo Torres por la identificación taxonómica de las especies animales. A los evaluadores que brindaron sugerencias para mejorar sustancialmente la publicación. Este trabajo fue posible desarrollar por los aportes económicos del proyecto "Percepción y uso de flora y fauna por parte de los pobladores del sistema Laguna Mar chiquita-Bañados del Río Dulce: diagnóstico e implicancias para la conservación", financiado por el Ministerio de Ciencia y Técnica del Gobierno de la Provincia de Córdoba.

\section{REFERENCIAS}

Aguilera, RM; A DURAND-SMith; EM RodríGueZ \& M Romero MENDOZA. 2003. Veinticinco años de investigación cualitativa en salud mental y adicciones con poblaciones ocultas. Salud Mental, 26(6):76-83.

Altricher, M. 2006. Wild life in the life of local people of the semi-arid Argentine Chaco. Biod. and Conser., 15:27192736.

ARENAS, P. 2003. Etnografía y alimentación entre los Toba-Ñachilamol ek y Wichí-Lhuku'tas del Chaco Central: Argentina. Buenos Aires, Argentina. Pp. 562.

ArenAs, P \& G Porint. 2009. Las aves en la vida de los tobas del oeste de la provincia de Formosa (Argentina). Tiempo de Historia, Asunción, Paraguay. Pp. 300.

Arias Toledo, B; C Trillo \& M Grilli. 2010. Uso de plantas medicinales en relación al estado de conservación del bosque en Córdoba, Argentina. Ecolog. Aust., 20:235-246.

Arias Toledo, B; C Trillo; M Grilli; S Colantonio \& L Galetto. 2014. Relationships between land-use types and plant species used by traditional ethno-medical system. European J. of Med. Plants, 4(9):998-1021.

ARIAS Toledo, B \& C TRILlo. 2014. Animales y plantas que curan: avances sobre la farmacopea natural de los pobladores del área de Laguna Mar Chiquita. Revista de la Facultad de Ciencias Exactas Físicas y Naturales, 1(2):77-85.

BARBARÁn, FR. 1999. Comercialización de cueros de pecarí (Tayassu spp.) en el Chaco semiárido de la Provincia de Salta, Argentina, período 1973-1997. Pp. 195-206 in: Fang,TG; OL Montenegro \& RE Bodmer (eds.). Manejo y Conservación de Fauna Silvestre en América Latina. Instituto de Ecología, La Paz, Bolivia.

Barbarán, F. 2003.Factibilidad de Caza de Subsistencia, Comercial y Deportiva en el Chaco Semiárido de la Provincia de Salta, Argentina. Fermentum, 36:89-117.

BERDUC, A. 2004. Acerca de los usos de la fauna nativa en el Cerro Colorado. Una aproximación. Cartilla de divulgación de trabajo realizado en el marco de una Beca de la Secretaría de Extensión Universitaria, Universidad Nacional de Córdoba. Argentina.

BERKES, F. 1999. Sacred Ecology - Traditional ecological knowledge and resource management. Taylor \& Francis, Filadelfia, EE.UU. Pp. 209.

CaVANna, C; R CASTRO; R CoIrINI; U Karlin \& M Karlin. 2009. Caracterización socio-productiva de ocho comunidades de pequeños productores de las Salinas Grandes, provincia de Catamarca, Argentina. Multequina, 18:15-29.

Corcuera, R. 2006. Mujeres de seda y tierra. Ed. Argentina, Bs.As

Costa Neto, EM. 2002. Manual de Etnoentomología. M\&T-Manuales \& Tesis SEA. CYTED, ORCYT-UNESCO \& SEA (eds.). Pp. 104

CunNINGHAM,AB.2001. Etnobotánicaaplicada.Pueblos, usodeplantassilvestresyconservación.WWF-UK,UNESCO,Royal Botanic Gardens, Kew, United Kingdom. Ediciones Nordan Comunidad, Montevideo, Uruguay.

CURTO, E. 2009. Selección de relictos de bosque comolugares de valor especial para conservación en la Reserva MarChiquita, Córdoba, Argentina. Tesis de Maestría del Programa de Post grado en Manejo de vida silvestre, Centro de Zoología aplicada, FCEFyN, Universidad Nacional de Córdoba. Argentina.

Di Lullo, O. 1946. Contribución al estudio de las Voces Santiagueñas. Gobierno de la Provincia de Santiago del Estero, Argentina.

Didac Santos-Fita, E; J Naranjo \& JL Rangel-Salazar. 2012. Wildlife uses and hunting patterns in rural communities of the Yucatan Peninsula, Mexico. J. of Ethnob. and Ethnomed., 8:38.

Eyssartier, C; AH LAdio \& M LozAda. 2009. Uso de plantas medicinales cultivadas en una comunidad semi-rural de la estepa patagónica. BLACMA, 8(2):77-85.

FAO. 2011. Situación de los bosque. El valor local de los bosques. Cap. 4. ONU. Roma, Italia.

GIRAUDO, A. 2009. Defaunación como consecuencia de las actividades humanas en la llanura del Chaco argentino. En: El Chaco sin bosques: la Pampa o el desierto del futuro. Morello, JH \& A Rodríguez (eds.). Orientación Gráfica Ed. Bs. As., Argentina.

Haro, JG; P Michelutti; RM Torres; A Molli \& E Bucher. 2006. Mamíferos. Pp. 191-199 en: Bucher, EH (ed.). Bañados del Río Dulce y laguna Mar Chiquita (Córdoba, Argentina). Academia Nacional de Ciencia, Córdoba, Argentina.

KARPPINEN, H. 1998. Values and objectives of non-industrial private forest owners in Finland. Silva Fennica, 32(1):4359.

Majumdar, I; L Teeter \& B ButLer. 2008. Characterizing family forest owners: a cluster analysis approach. Forest Science, 54(2):176-184. 
Matlin, M \& H Foley. 1996. Sensación y Percepción. Prentice Hall. México. Pp. 554. Medrano, MC \& CN Rosso. 2012. Tigres, ñandúes y otras mercancías. Aprovechamiento de fauna entre grupos guaicurúes en el gran chaco argentino durante el Siglo XVIII. Bicentenario: Revista de Historia de Chile y América, 11:1-25.

Menghi, M. 2006. Vegetación. En: Bucher, EH (ed.). Bañados del Río Dulce y laguna Mar Chiquita (Córdoba, Argentina). Academia Nacional de Ciencia, Córdoba, Argentina.

NAVARRo, JL; VR Rosati \& EC FraIRE. 1997. Incidencia de vizcachas (Lagostomus maximus) en un cultivo de soja. Mastozoo Neotrop., 4(2):137-144

Pautasso, A. 2003. Aprovechamiento de la fauna silvestre por pobladores rurales en la fracción norte de los bajos submeridionales de la provincia de Santa Fe, Argentina. (Incluye aspectos relacionados a la producción y la conservación en este ambiente. Com. Mus. Prov. Cs. Naturales "Florentino Ameghino", 1-62.

RíosVILLAMIL,JCA.2013. EstructurapoblacionaldecomunidadesaledañasalalagunadeMarChiquita.Pautasmatrimonialesy efectos de la inmigración de principios del siglo XX. Tesina para acceder al título de Biólogo, Facultad de Ciencias Exactas Físicas y Naturales Universidad Nacional de Córdoba- Argentina.

ROSACHER, C. 2011. Cartilla informativa para la conservación de nuestra fauna silvestre. Secretaria de Ambiente, Gobierno de la provincia de Córdoba. Argentina.

ToRres, RM \& P Michelutti. 2006. Aves acuáticas. En: Bucher, EH (ed.). Bañados del Río Dulce y laguna Mar Chiquita (Córdoba, Argentina). Academia Nacional de Ciencia, Córdoba, Argentina.

TRILLO, C. 2010. Valoración del bosque y uso delas plantas silvestres por partedelos pobladores dela Sierra de Guasapampa, Noroeste de la Provincia de Córdoba. Tesis Doctoral, Facultad de Ciencias Exactas Físicas y Naturales, Universidad Nacional de Córdoba. Argentina.

TRILlo, C; S Colantonio \& L Galetto. 2014. Perceptions and use of native forests in the arid Chaco of Córdoba, Argentina. Ethnobot. Resear. and Appl., 12:497-510. 